\title{
Elaborative processing and conjunction errors in recognition memory
}

\author{
JASON ARNDT \\ Middlebury College, Middlebury, Vermont \\ AND \\ TODD C. Jones \\ Victoria University, Wellington, New Zealand
}

\begin{abstract}
Four experiments were conducted in order to examine the influence of elaborative processing at encoding on recognition memory conjunction lure errors. In these experiments, participants generated cues for compound words as wholes (e.g., haywire) or as separate entities (e.g., hay, wire). Studied words were re-presented in exact form (old) or recombined to form conjunction lures on the recognition test. Participants were asked to make old new judgments and to indicate whether they had rejected items judged to be new because of recall of a studied item or because of lack of familiarity with an item. The results suggested that recall-to-reject processing and conjunction lure familiarity increased with both types of generation, although generation of cues for compound words as a whole did not influence conjunction lure error rates. An emphasis on processing each constituent of a compound word during encoding increased the familiarity of those constituents more than generation of a compound word as a whole, resulting in an increase in conjunction lure errors. These results suggest that both familiarity and recollection-based monitoring processes influence conjunction lure errors, and therefore support dual-process theories of recognition memory.
\end{abstract}

In recent years, there has been a substantial increase in research devoted to the study of memory errors in listlearning paradigms (see Roediger, McDermott, \& Robinson, 1998, for a review). A fundamental question concerns the kinds of processes that produce and limit these errors. The present study addresses the extent to which a relatively automatic process, familiarity, induces errors and to which a controlled process, recollection, can be used to reduce errors, a retrieval strategy often referred to as recall-to-reject or recollection rejection (Brainerd, Reyna, Wright, \& Mojardin, 2003; Hintzman, Curran, \& Oppy, 1992; Jacoby, 1991; Rotello, Macmillan, \& Van Tassell, 2000). We employed the memory conjunction paradigm (Reinitz, Lammers, \& Cochran, 1992; Underwood, Kapelak, \& Malmi, 1976; Underwood \& Zimmerman, 1973 ) in order to study the contributions of familiarity and of recollection to errors in recognition memory. In this paradigm, participants study compound words, such as haywire and smokestack, which are referred to as parents. On a subsequent memory test, participants make recognition memory judgments for words encountered in the study list; nonstudied lures that are formed by combining components of parent items, such as haystack, which are referred to as conjunction lures; and entirely new words.

Substantial evidence supports recognition memory theories that include both familiarity and recollection pro- cesses (i.e., dual-process theories; Diana, Reder, Arndt, \& Park, 2006). For the conjunction paradigm, dual-process theories propose that conjunction errors arise due to the familiarity of the constituents that make up a conjunction lure (Jones \& Jacoby, 2001), which produces a false sense of prior experience for conjunction lures. A dual-process explanation also advances the idea that the familiarity that underlies conjunction lures can be counteracted by the successful recall of parent words (i.e., recall-to-reject processing; Jones \& Atchley, 2006; Jones \& Jacoby, 2001; Lampinen, Odegard, \& Neuschatz, 2004; Odegard, Lampinen, \& Toglia, 2005). Thus, conjunction errors have been characterized as reflecting the influence of familiarity in the absence of recollection for the parent words. If recollection for the parent words is at or near zero, the conjunction error rate provides a good measure of familiarity engendered by combining parents' components. However, to the extent that parent words are recollected when conjunction lures are tested, the conjunction error rate will underestimate conjunction lure familiarity.

In contrast with the antagonistic relationship that recollection and familiarity have when one is assessing conjunction lures, dual-process theory suggests that the two processes work in concert when evaluating studied items, so that a studied item can be endorsed on the basis of familiarity from or recollection of its occurrence during a

J.Arndt, jarndt@middlebury.edu 
study list (Jones \& Jacoby, 2001). What this arrangement predicts is that manipulations that increase recollection should increase hit rates and decrease conjunction lure errors, provided that conjunction lures are able to cue recollection of one of their parents as often as parent words are able to cue recollection of their own occurrence in a study list. However, the cues for recalling a parent item when tested with a conjunction lure are typically poor because there is relatively little semantic overlap between parent items and conjunction lures (Jones \& Jacoby, 2001; Odegard et al., 2005). Thus, the influence of recollection to dampen conjunction lure errors is likely to be weaker than the influence of recollection to boost old-item recognition. In fact, several studies have found that in relation to the hit rate, the conjunction error rate is less sensitive to study manipulations that are thought to influence recollection, such as study-item repetition (Jones, 2005; Jones \& Jacoby, 2001), level of processing (Jones, Brown, \& Atchley, 2007), and divided versus full attention (Jones \& Jacoby, 2001). Indeed, the typical pattern of results from such studies has shown a dissociation such that hits are affected by study manipulations, but conjunction errors are not (Jones et al., 2007; Jones \& Jacoby, 2001; Reinitz, Verfaellie, \& Milberg, 1996).

Given the tendency for conjunction error rates to be unaffected by study manipulations, it is difficult to determine how or whether familiarity and recall-to-reject are influenced by encoding conditions. In response to this difficulty, recent approaches have scrutinized the basis for correct rejections of conjunction lures (Jones, 2005; Jones \& Atchley, 2006; Lampinen et al., 2004; Odegard et al., 2005). Three measures have been used, sometimes in combination. One measure utilizes characteristics of receiver operator characteristic (ROC) curves; recall-toreject processing will cause the ROC that plots conjunction lures against new items to fall below the major diagonal on probability ROC plots (Lampinen et al., 2004). A second measure requires participants to judge the memorial basis they used to reject test items (Jones, 2005; Jones $\&$ Atchley, 2006). Specifically, participants are asked to indicate whether they rejected a test item based on recall of a studied item - a measure of recall-to-reject processing - or based on lack of familiarity. The third approach is to solicit actual recall of parent words, either during (e.g., Jones \& Atchley, 2006) or after (Lampinen et al., 2004) the recognition test (see Gallo, 2004, for a similar procedure in the Deese/Roediger-McDermott paradigm). Whereas recall of the actual parent words provides the most direct evidence of recall-to-reject processing, recall judgments and actual recall generally produce similar estimates of recall-to-reject processing and are affected similarly by experimental manipulations (Jones \& Atchley, 2006).

Using recall judgments, Jones (2005) found that although conjunction lure error rates were not influenced by changes in study repetition, judgments of recall-to-reject processing increased with study repetition. Thus, even when encoding conditions did not affect conjunction lure errors, it was shown that the study conditions increased recall-to-reject processing. Direct measures of recall-toreject can reveal improved recall-to-reject processing, even when conjunction errors increase. For example, conjunction lures that semantically overlap with a parent word (e.g., the conjunction lure handgun constructed from parent words handball and shotgun) have produced a higher conjunction error rate than conjunction lures that do not semantically overlap with a parent word (e.g., the conjunction lure blackbird constructed from the parent words blackmail and jailbird; Odegard et al., 2005). Nevertheless, strong semantic parent-to-conjunction overlap also increased recall of parent words. Both of these results underscore the importance of directly measuring recallto-reject, because they suggest that conjunction error rates do not necessarily provide a complete or accurate picture of recall-to-reject processing (Odegard et al., 2005).

By obtaining direct measures of recollection, it is possible to gain leverage on a second issue: measuring familiarity for conjunction lures. Any measure of familiarity derived from dual-process theory depends on the assumed relationship of familiarity and recollection. We considered three possible relationships that have been assumed by dual-process theorists: independence (Yonelinas \& Jacoby, 1995), redundancy (Joordens \& Merikle, 1993), and mutual exclusivity (Gardiner, 1988). All of these models have been used either implicitly or directly to derive process estimates of recollection and familiarity from remember-know judgments. We applied the reasoning from remember-know studies for items judged to be old to the present studies, in which we collected direct measures of recall-to-reject processing and familiarity in the absence of recall-to-reject for conjunction lures. For all three models, recollection was measured by the participants' ability to recall a parent item when presented with a conjunction lure. The conjunction error rate provides an index of familiarity, but it is incomplete for two of the relationships: independence and redundancy. For the independence model, the measure of familiarity takes into account how often recollection and familiarity may have co-occurred, so the basic equation for measuring conjunction lure familiarity is as follows: familiarity $=$ conjunction error rate/( 1 - recall rate). For the redundancy model, items that are recollected are necessarily familiar, so familiarity $=$ conjunction error rate + recall rate. Finally, for the mutual exclusivity model, the measure of familiarity is simply the conjunction error rate, because this model assumes that familiarity and recollection never co-occur.

There are two potential concerns that arise from using the conjunction lure paradigm to understand the relationship between recollection and familiarity. First, it may seem that the use of recall to reject conjunction lures necessitates that the lures also be familiar. If this were the case, testing the mutual exclusivity model would be unreasonable, because the model would be fundamentally incompatible with the nature of the processes underlying conjunction lure errors. However, theoretical models of recollection and familiarity (e.g., Diana et al., 2006; Jacoby, 1991; Reder et al., 2000) generally assume that the use of recollection is prioritized over the use of familiarity, because recollection is the more reliable and certain basis for determining whether a test item was studied. Similarly, because recall-to-reject processing is a certain basis for 
rejecting conjunction lures, it is likely to be the preferred strategy for assessing the lures' studied status. If recall-toreject fails, participants may use familiarity as a basis for evaluating conjunction lures. The important point for our purposes is that conjunction lures need not be familiar in order for recall-to-reject to occur, because recollection, and therefore recall-to-reject, is generally given priority when making recognition judgments, and familiarity will be consulted only in the absence of recall-to-reject. ${ }^{1}$

A second concern is that the measures of recollection and familiarity we will use to understand the recollectionfamiliarity relationship are based on different items (recall of parent items and conjunction lure errors, respectively). However, in the conjunction lure paradigm, the cuing of familiarity and recall-to-reject are both based on the encoded representation of parents and are both cued by the same elements of conjunction lures at test. Specifically, the familiarity that produces conjunction lure errors arises from the fact that the two components in conjunction lures are encoded as part of the lure's parents during study. Similarly, the recall of parents as a basis for rejecting conjunction lures is cued by the presence of a component of a studied parent item in a conjunction lure at test. Thus, measurement of recollection and familiarity in the conjunction lure paradigm is directly comparable to a standard recognition memory experiment in which a test item could be recognized on the basis of the recollection of its occurrence in a study list or because the test item was familiar but its occurrence in the study list was not recollected.

In sum, the prior literature provides evidence that dualprocess models of recognition can be applied in order to understand performance in the conjunction lure paradigm. Further, recent work has demonstrated that conjunction error rates often fail to reflect changes in recall-to-reject processing across experimental conditions. This could lead to inaccurate conclusions about the roles that familiarity and recollection play in increasing and decreasing conjunction lure errors, unless a direct measure of recallto-reject processing is collected. Finally, relying on conjunction errors as a full measure of familiarity implicitly assumes that recollection and familiarity are mutually exclusive processes. Thus, such reliance may underestimate conjunction lures' familiarity levels if recollection and familiarity are indeed independent or redundant processes.

In the present experiments, we examined the extent to which elaborative encoding of parent words or the components of parent words affected conjunction lure errors and recall-to-reject processing. In Experiments 1A and 1B, we compared a condition in which participants copied parent words at study to conditions in which participants copied the parent words and generated either one or three cues for it. In Experiments $2 \mathrm{~A}$ and $2 \mathrm{~B}$, we compared a condition in which participants copied parent words at study with conditions in which they generated cues either for a parent word as a whole or for the separate components of a parent word. We chose study manipulations that stressed elaborative processing because generation and levels of processing have been shown to influence recollection more than they influence familiarity (see Yonelinas, 2002, for a meta-analysis and review), making elaborative pro- cessing a promising candidate to produce large increases in recall-to-reject processing that were not offset entirely by increases in familiarity. We collected direct measures of recall-to-reject processing by asking participants to indicate when they used recall of a studied item to reject a test item. Finally, we verified participants' judgments of recall-to-reject processing by asking them to produce the parent item that was used as a basis for their judgment. Thus, we were able to generate an objective record of recall-to-reject processing, which could then be used to understand the contribution of recollection and familiarity to conjunction lure errors.

Four prior studies provide relevant data with regard to the effects of elaborative encoding on conjunction error rates. Ghatala, Levin, Bell, Truman, and Lodico (1978) manipulated the semantic relationship of study words (e.g., bed, spread) to conjunction lure words (bedspread) by accompanying each study item with a prime that biased the interpretation of the parents toward or away from the meaning of the conjunction lure. They found that study items presented in a semantic context that was biased toward the meaning of the conjunction lure (e.g., soft for bed, cloth for spread) increased the likelihood of a conjunction lure error, in comparison with study items presented in a semantic context that was biased away from the meaning of the conjunction lure (e.g., river for bed, cheese for spread). Odegard et al. (2005) compared conjunction lure errors for which the meanings evoked by the parent words (e.g., handball, shotgun) strongly overlapped that of the conjunction lure (e.g., handgun) with those for which the meanings evoked by parents (e.g., blackmail, jailbird) failed to overlap that of the conjunction lure (e.g., blackbird). Odegard et al. found that this manipulation either did not influence conjunction lure errors (Experiment 1) or led to more errors when parents and conjunction lures shared meanings (Experiment 2). Finally, in two studies, researchers manipulated the level of processing (shallow vs. deep) during study (Jones et al., 2007; Reinitz et al., 1996), and both found that a deep level of processing increased hit rates, and conjunction error rates were unaffected.

Given that none of these prior studies obtained a decrease in conjunction error rates with a corresponding increase in hit rates, we had no basis for predicting whether generating properties for parent items would increase recall-to-reject processing enough to reduce conjunction error rates in comparison with a control condition. If anything, these prior studies suggest that conjunction errors should be higher in conditions that stress semantic processing (Ghatala et al., 1978; Odegard et al., 2005, Experiment 2). However, because changes in conjunction error rates do not appear to provide an accurate assessment of recall-to-reject processing, it seems possible that recall-to-reject processing could increase across the same conditions in which conjunction error rates are invariant (Jones et al., 2007; Reinitz et al., 1996), or even when conjunction lure errors increase (Odegard et al., 2005). Thus, collecting recall-to-reject judgments in the present study allowed us to better understand how elaborative encoding influenced recall-to-reject processing and familiarity for conjunction lure errors without regard to whether 
conjunction lure errors increased, decreased, or remained invariant across encoding conditions.

\section{EXPERIMENTS 1A AND 1B}

In Experiments 1A and 1B, we implemented elaborative encoding conditions that required participants to generate cues for whole parent items. We used encoding conditions in which participants were asked to produce zero, one, or three cues for each parent word. Mäntylä (1986) and Mäntylä and Nilsson (1983) demonstrated the substantial benefit of generating cues for study words during encoding on later recall. As a result, it is likely that this study manipulation will produce high levels of parent word recollection, which will enhance the opportunity to observe differences in the ability to use recall of parent items to reject conjunction lures.

Evidence that generation at encoding can enable memory editing processes has recently been reported (Jones, 2006). In Jones's Experiment 1, participants generated some conjunction stimuli from parent words during the study task, but did not generate others. On a subsequent recognition test, participants were instructed to exclude all conjunction stimuli from old judgments without regard to whether or not they had been generated during the study phase. Under a short response signal delay (RSD) test procedure, generated conjunction words produced a higher error rate than did nonstudied conjunction lures; but under a long RSD procedure, generated conjunction words produced a lower error rate than did nonstudied conjunction lures. These results suggest that relative to nonstudied conjunction lures, study generation of the conjunction lures increased familiarity, which was evidenced by the higher error rate in the short RSD condition, as well as recollection, evidenced by the lower error rate in the long RSD condition. The present experiments further investigated the extent to which generation at study enabled memory editing processes. This was done by assessing whether the generation of cues related to parent words (rather than the direct generation of conjunction lures, studied by Jones, 2006) also improved the ability to reject conjunction lures, due to recall of their parent words.

Minor differences for Experiments $1 \mathrm{~A}$ and $1 \mathrm{~B}$ included the participants, slightly different item sets, and the test instructions and procedures. The participants were in different countries (New Zealand, U.S.A.); thus the materials were adjusted slightly to be appropriate for New
Zealanders and Americans. The test instructions and procedures for both experiments included standard old-new recognition memory instructions and procedures. For Experiment $1 \mathrm{~B}$, additional instructions and responses were solicited in order to assess the use of recall-to-reject processing. For all items judged to be new, participants were asked to indicate whether they had reached that judgment because they recalled a studied, related parent word (a recall response) or because the test item was not familiar (a know response). Furthermore, if a test item was judged to be new because of recall, the participants were asked to recall the parent word they had used as the basis for the recall response (Jones, 2005; Jones \& Atchley, 2006). The rationale for this pairing of studies was to ensure that conjunction lure error rates were comparable when participants were asked simply to discriminate old and new items and when they were asked to judge the basis for rejecting a test item.

\section{Method}

Participants. Twenty-four students at Victoria University of Wellington participated in Experiment 1A, and 24 students at Middlebury College participated in Experiment 1B.

Materials. The stimulus materials were 72 compound-word triplets taken from Jones (2005). An additional 6 compound words were used as primacy and recency buffers. Each triplet was composed of 2 parent words (e.g., watchtower, sheepdog) and 1 offspring word (e.g., watchdog), which was created by combining the first portion of a parent word with the second portion of the other parent word. None of the lexical-morpheme constituents of the compound words (parents, offspring) were repeated across triplets. Sheets of paper (A4) were supplied to the participants for the study phase. The sheets had 114 numbered lines with four blanks next to each numeral.

For all experiments reported in this article, computer programs were composed using E-Prime software (Schneider, Eschman, \& Zuccolotto, 2002), and the programs were run on IBM-compatible computers interfaced with VGA monitors. Furthermore, in all experiments reported in this article, only parent words were presented during encoding, whereas both parent items and offspring were presented during the recognition memory test. Finally, the method for constructing item triplets (two parents and one offspring) was identical in all experiments.

Design and study-test list construction. Table 1 illustrates the basic design of Experiments 1A and 1B. A 2 (test item type: parent words vs. offspring) $\times 4$ (study task: copy vs. generate one cue word vs. generate three cue words vs. nonstudied) within-participants design was employed. The 72 word triplets were assigned to one of eight stimulus sets, with 9 word triplets in each set. Each of the eight stimulus sets was assigned to one of the experimental conditions formed by the combination of test item type and study task. Thus, parents from six of the stimulus sets were shown at study, whereas

Table 1

Schematic of Study and Test Conditions With Example Stimuli for Experiments 1A and 1B

\begin{tabular}{|c|c|c|c|}
\hline Study Word & Study Task & Test Word & Test Item Type \\
\hline blackmail & copy & blackmail & old (parent) \\
\hline lawsuit & generate 1 cue & law & old (par \\
\hline nightmare & generate 3 & nig & old (par \\
\hline - & & pas & nt baseline) \\
\hline crossroad, rainbow & copy & cros & conjunctio \\
\hline watchtower, sheepdog & generate 1 cue & watchdog & conjunction lure \\
\hline switchblade, billboard & generate 3 cues & $\begin{array}{l}\text { switchboard } \\
\text { shortcut }\end{array}$ & $\begin{array}{l}\text { conjunction lure } \\
\text { new (conjunction baseline) }\end{array}$ \\
\hline
\end{tabular}


parents or offspring from all eight stimulus sets were shown in the test phase. The eight stimulus sets were rotated through the eight experimental conditions formed by the crossing of test item type and study task, which ensured that each set occurred equally often in each of the experimental conditions across participants.

For Experiment 1A, a single study order based on test item type was constructed so that no more than two items from the same level of test item type were presented in succession. There were 108 study trials containing 54 parent words to be presented on the recognition memory test and 54 study primes for the conjunction lures. Three primacy and three recency buffers were included in the study list, with one compound word from each of the primacy and recency buffers assigned to each of the study tasks, producing an overall study list length of 114 words. For Experiment 1B, studied parent items were presented entirely randomly, with the exception of the items designated as primacy and recency buffers.

The test list for each experiment included the studied parent words from half of the stimulus sets presented during the study list (54 items total: one third from the copy study condition, one third from the one-cue-generated study condition, and the final third from the three-cues-generated study condition); the conjunction lures corresponding to the other half of the stimulus sets presented during the study list ( 27 items total: one third from the copy study condition, one third from the one-cue-generated study condition, and the final third from the three-cues-generated study condition); the parent words from one of the nonstudied stimulus sets (18 items); and the conjunction lures from one of the nonstudied stimulus sets (nine items), yielding a test list length of 108 words.

Procedure. In the study phase, all participants were instructed to read each of the study words presented on a computer screen in preparation for a subsequent memory test. The participants were directed to follow any written instructions that appeared below the study word. On each study trial, a word was displayed for $3 \mathrm{sec}$, followed by instructions below the word, which prompted the participant to write the study word itself (e.g., daredevil), write the study word plus one self-generated word as a cue to the study word (e.g., daredevil, followed by adventurous), or write the study word plus three self-generated words as cues (e.g., daredevil, followed by $\mathrm{ad}$ venturous, stuntman, crazy). Up to $30 \mathrm{sec}$ was given for participants to accomplish the writing aspect of each trial, but the participants were able to start the next trial before reaching the 30 -sec time limit by pressing the "N" key. There was a 250 -msec intertrial inverval.

The recognition memory test commenced immediately after the study phase. In the recognition memory test instructions, participants were warned about the presence of conjunction lures, given an example of a conjunction lure and its parent words, instructed about the difference between them, and asked to avoid conjunction lure errors. Items were presented in a random order at test. In Experiment 1A, participants were asked to make a single old-new recognition judgment for each test item. In Experiment 1B, participants were prompted to make up to three judgments for each word presented on the recognition memory test. The first prompt asked participants to indicate whether a test item was old or new, which replicated the judgment required in Experiment 1A. The second prompt asked participants to indicate why they judged a test item to be new-due to recall of a parent item (recall), or due to the test item being unfamiliar (know). The third prompt asked participants who indicated having judged an item to be new due to recall of a parent item to type the parent item into a response box.

\section{Results}

The baseline false alarm rate was similar for parent words $(M=.04)$ and offspring $(M=.06)$ in Experiment $1 \mathrm{~A}$, as well as in Experiment $1 \mathrm{~B}(M=.10$ for both parents and offspring). Corrected parent-word recognition scores and conjunction lure error rates were computed by subtracting the baseline false alarm rates from the parent-word recognition hit rates and conjunction lure false alarm rates. ${ }^{2}$
Corrected recognition scores are displayed in Figure 1 for Experiment 1A (top panel) and Experiment 1B (bottom panel). Hits, false alarms to conjunction lures, and false alarms to baseline items are presented in Table 2 .

As can be seen in Figure 1, the two experiments produced very similar results: Generating cues for parent words during the study phase produced a dramatic increase in parent word recognition but had a negligible effect on conjunction lure errors. A 3 (study task) $\times 2$ (test item type) repeated measures ANOVA on the corrected recognition scores confirmed this interaction [Experiment $1 \mathrm{~A}, F(2,46)=24.88, M S_{\mathrm{e}}=.011$; Experiment $1 \mathrm{~B}$, $\left.F(2,46)=28.04, M S_{\mathrm{e}}=.011\right]$. The main effects of study task [Experiment $1 \mathrm{~A}, F(2,46)=36.77, M S_{\mathrm{e}}=.012$; Experiment $\left.1 \mathrm{~B}, F(2,46)=28.21, M S_{\mathrm{e}}=.012\right]$ and test item type [Experiment 1A, $F(1,23)=361.24, M S_{\mathrm{e}}=.048$; Experiment $\left.1 \mathrm{~B}, F(2,46)=426.87, M S_{\mathrm{e}}=.042\right]$ were also
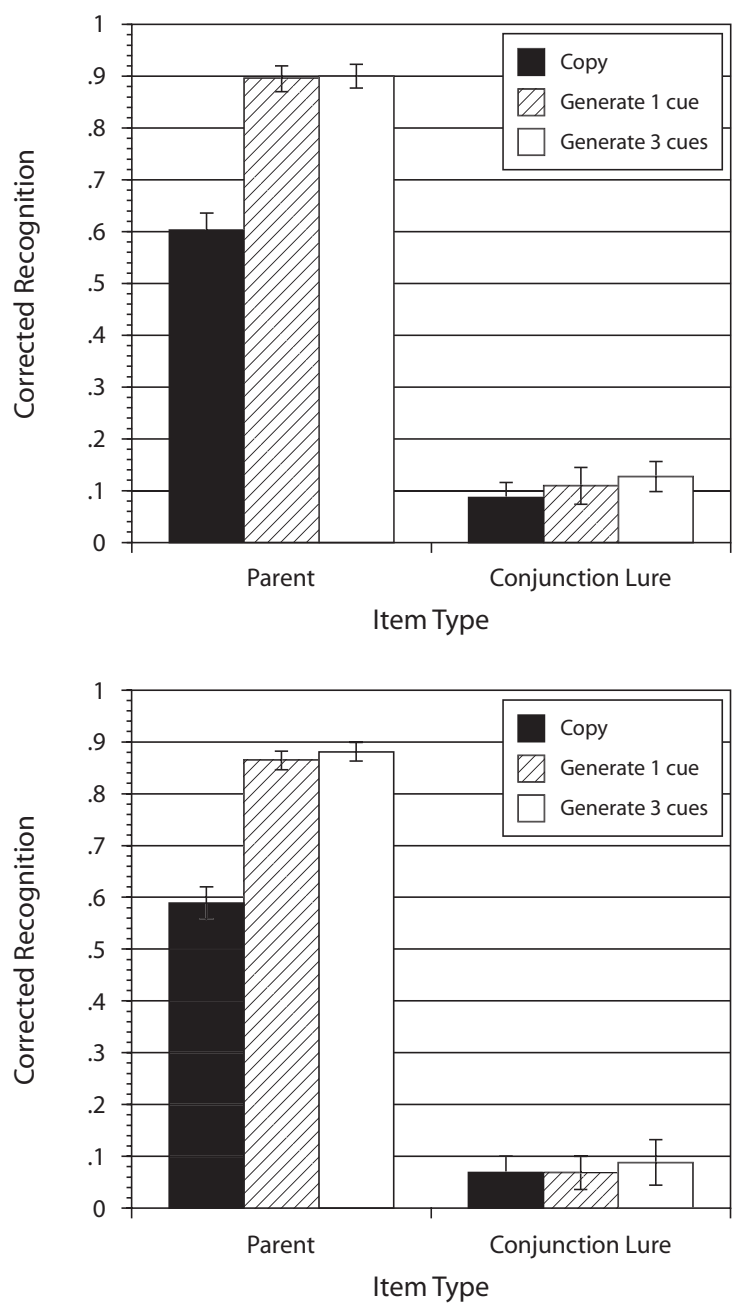

Figure 1. Parent word and conjunction lure recognition, as a function of study task in Experiment 1A (top panel) and Experiment 1B (bottom panel). Error bars depict the standard errors of the means. 
significant. To explore the interaction, separate repeated measures ANOVAs were conducted on parent word recognition and conjunction lure errors using study task as the experimental factor. These analyses produced a reliable effect of study task for parent word recognition [Experiment 1A, $F(2,46)=76.98, M S_{\mathrm{e}}=.009$; Experiment 1B, $\left.F(2,46)=66.97, M S_{\mathrm{e}}=.010\right]$, but not for conjunction lure errors [Experiment 1A, $F(2,46)=0.71, M S_{\mathrm{e}}=.014$; Experiment 1B, $\left.F(2,46)=0.21, M S_{\mathrm{e}}=.013\right]$. Bonferroniadjusted comparisons showed that each of the two study conditions for which a cue was generated produced significantly higher parent word recognition than did the copy condition. The two cue conditions were not significantly different from one another, although performance was close to ceiling in both conditions. Thus, whether the generation of multiple cue words provides an additional benefit to parent word recognition cannot be ascertained from the present data. Mäntylä's (1986) results, however, did show a benefit of generating multiple cue words during the study phase. But the pattern of central importance from these two experiments is that despite the very large jump in accuracy of parent word recognition in the cue generation conditions, this advantage was not associated with fewer conjunction lure errors. Indeed, if anything, the pattern of the means for conjunction lure errors suggests that conjunction lures produced higher levels of false memory in the three-cues-generated condition than in the copy condition.

Recall responses. Because the conjunction error rates were not affected by encoding conditions in Experiments $1 \mathrm{~A}$ and $1 \mathrm{~B}$, they do not provide a strong indication of how or whether recall-to-reject processing was influenced by elaborative encoding conditions. However, the recall judgments and actual recall from Experiment 1B provide a more complete picture of the processes engaged during retrieval. Before turning to the data of interest, it is important to note that the probability of making a recall response to items for which no competing episodic memory existed was low. Recall responses to parent items (largest $M=.04$ in the copy study condition), as well as new items that served to measure baseline error rates for both parent items $(M=.06)$ and conjunction lures $(M=$ .05 ), were rare. Furthermore, the conditional probability of making a recall response, given that a conjunction lure was called new, was greater than $50 \%$, whereas the conditional probability of making a recall response, given that any other test item was called new, was less than $9 \%$. Thus, when participants' memory was tested for old or unstudied items, they largely deemed those items to be new based on the lack of familiarity; that is, they gave a know response. In comparison, participants regularly reported the use of parent item recall as a basis for rejecting conjunction lures, suggesting that they were able to use recall and know judgments accurately.

The probability of rejecting a conjunction lure due to the self-judged recall of a parent item and the probability of actually recalling a parent item when tested with a conjunction lure are presented in Figure 2, as a function of study task. Participants were able to use recall-to-reject processing to judge lure items correctly as being new more
Table 2

Hits for Parent Items, False Alarms for Conjunction Lures, and False Alarms for Baseline Items in Experiments $1 \mathrm{~A}$ and $1 \mathrm{~B}$

\begin{tabular}{|c|c|c|c|c|c|c|c|c|}
\hline & \multicolumn{2}{|c|}{ Copy } & \multicolumn{2}{|c|}{$\begin{array}{l}\text { Generate } \\
1 \text { Cue }\end{array}$} & \multicolumn{2}{|c|}{$\begin{array}{c}\text { Generate } \\
3 \text { Cues } \\
\end{array}$} & \multicolumn{2}{|c|}{ New } \\
\hline & $M$ & $S E$ & $M$ & $S E$ & $M$ & $S E$ & $M$ & $S E$ \\
\hline \multicolumn{9}{|c|}{ Experiment 1A } \\
\hline Parent & .64 & .03 & .94 & .01 & .94 & .01 & .04 & .02 \\
\hline Conjunction lure & .15 & .03 & .17 & .04 & .19 & .03 & .06 & .02 \\
\hline \multicolumn{9}{|c|}{ Experiment 1B } \\
\hline Parent & .69 & .03 & .97 & .01 & .98 & .01 & .10 & .02 \\
\hline Conjunction lure & .17 & .03 & .17 & .03 & .19 & .04 & .10 & .03 \\
\hline
\end{tabular}

often in the one-cue-generation condition than in the copy condition, and more often in the three-cue-generation condition than in the one-cue-generation condition. Furthermore, the ability to produce a parent item in order to justify a recall response generally supported the validity of recall judgments (Jones, 2005; Jones \& Atchley, 2006). Although the probability of accurately recalling a parent item as a basis for rejecting a conjunction lure was lower than the probability of a recall response, the pattern of performance across the study tasks was similar. These observations were supported by a reliable effect of study task on recall judgments and on actual recall [recall judgments, $F(2,46)=26.05, M S_{\mathrm{e}}=.023$; actual recall, $F(2,46)=$ $\left.21.64, M S_{\mathrm{e}}=.022\right]$. For both recall judgments and actual recall, Bonferroni-adjusted comparisons demonstrated that the one-cue-generation study condition produced a higher rate of recall-to-reject than did the copy condition, and the three-cues-generation condition produced a higher rate of recall-to-reject than either the copy condition or the one-cue-generation condition.

Familiarity underlying conjunction errors. Given that we collected measures of recall-to-reject processing and familiarity in the absence of recollection (the conjunction lure error rate), the data from these studies also

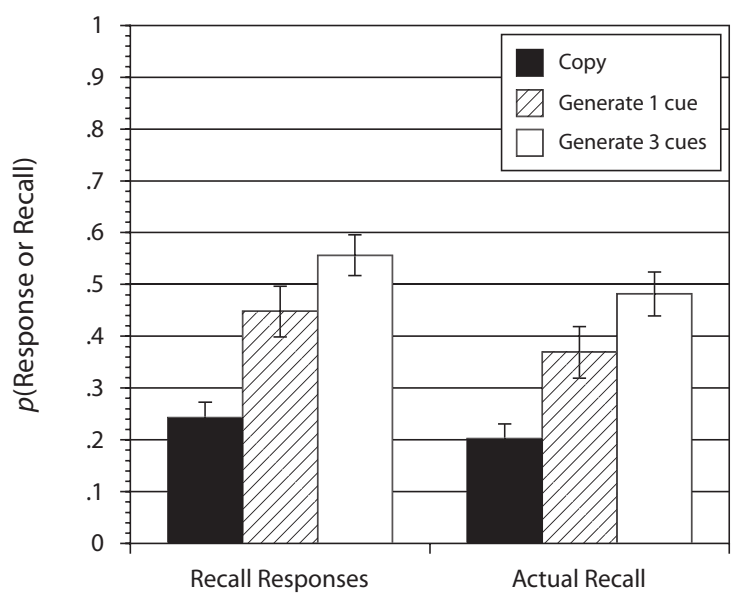

Figure 2. Recall responses and actual recall to conjunction lures judged to be new in Experiment 1B, as a function of study task. Error bars depict the standard errors of the means. 
allow us to estimate how conjunction lure familiarity was affected by the generation of cues for parent items. For three different assumptions about the relationship between recollection and familiarity (independence, redundancy, mutual exclusivity), the recall judgment rate or the actual recall rate provides an index of recollection. Since the actual recall rate is a bit more accurate, we used it instead of the recall judgment rate in our calculations of familiarity (see Table 3 ). Of central importance is the pattern of familiarity scores across the study conditions. The independence and redundancy approaches suggest that as more cues were generated for parent words, conjunction lure familiarity increased [independence, $F(2,46)=5.81$, $M S_{\mathrm{e}}=.08$; redundancy, $\left.F(2,46)=17.82, M S_{\mathrm{e}}=.06\right]$. Under the mutual exclusivity approach, the measure of familiarity is simply the conjunction error rate, which suggests the conclusion that familiarity was unaffected by an increase in elaborative processing at study, because conjunction errors were not influenced by study task.

\section{Discussion}

The results of these two experiments clarify the contribution of elaborative encoding to conjunction lure errors, as well as the use of strategic retrieval processes to limit conjunction lure errors. The results show that participants were better able to use recall-to-reject processing when they engaged in elaborative processing during encoding and that the more elaborative processing that was completed, the more recall-to-reject processing was used. Therefore, the finding that conjunction lure error rates were comparable across the three encoding conditions does not imply that familiarity and recall-to-reject processing were unaffected by elaborative encoding conditions. Instead, more sensitive measures indicate that elaborative encoding increased recall-to-reject processing. Within the context of dual-process theory, these two findings suggest that a second factor, such as familiarity, must have also increased with elaborative processing. Because dual-process theory views recall-to-reject and familiarity as antagonistic when judging conjunction lures, increased recall-to-reject processing should have produced lower conjunction lure error rates as more properties were generated if familiarity did not also increase with property generation. Thus, the data favor the view that familiarity is best measured by a model in which recollection and familiarity are either independent or redundant processes, because those two models suggested that familiarity increased with elaborative processing at encoding.

Table 3

Familiarity Estimates for Experiment 1B, Based on the Assumptions of the Independence, Redundancy, and Mutual-Exclusivity Models

\begin{tabular}{|c|c|c|c|c|c|c|}
\hline \multirow[b]{2}{*}{ Model } & \multicolumn{2}{|c|}{ Copy } & \multicolumn{2}{|c|}{$\begin{array}{c}\text { Generate } \\
1 \text { Cue }\end{array}$} & \multicolumn{2}{|c|}{$\begin{array}{l}\text { Generate } \\
3 \text { Cues }\end{array}$} \\
\hline & $M$ & $S E$ & $M$ & $S E$ & $M$ & $S E$ \\
\hline Independence & .17 & .03 & .24 & .04 & .35 & .06 \\
\hline Redundancy & .37 & .04 & .54 & .04 & .67 & .04 \\
\hline Mutual exclusivity & .17 & .03 & .17 & .03 & .19 & .04 \\
\hline
\end{tabular}

\section{EXPERIMENTS 2A AND 2B}

In Experiments 2A and 2B, we investigated how conjunction lure errors were influenced by the generation of a cue for each component of a compound parent word. Thus, instead of generating cues that referred to each studied parent word as a whole, participants sometimes generated cues that referred to each of the components that made up a parent word. For example, when presented with the word daredevil to study, they might generate truth (for dare) and angel (for devil). This manipulation therefore explored how elaboratively encoding the individual components of parent items influenced recall-to-reject processing and conjunction lure errors.

Prior research provides mixed evidence about how this manipulation should influence conjunction lure errors. Jones et al. (2007) found that studying components of later compound-word conjunction lures in isolation produced a lower conjunction error rate in comparison with a condition in which the components were studied as parts of compound words. However, Ghatala et al. (1978) found that when participants studied the components of conjunction lures in a context that biased interpretation toward the meaning of the conjunction lure, they committed more conjunction lure errors than in a condition in which the components of conjunction lures were studied in a context that biased interpretation away from the meaning of the lure. Finally, Odegard et al. (2005) showed that when conjunction lures were formed from parent words that shared meaning with them, recall-to-reject processing increased, although conjunction lures that shared meaning with parents still produced more errors than did conjunction lures formed from parent words that did not share meaning with them.

Given the variability in prior studies' findings, it is not clear whether generating properties for the components of parent items will increase or decrease conjunction lure errors. Furthermore, as reviewed in our introduction, it is possible for conjunction lure errors to increase (Odegard et al., 2005), decrease (Jones, 2006), or remain the same (Jones, 2005) as recall-to-reject processing increases. Thus, it is difficult to generate firm predictions about how generating cues for the components of parent items should influence familiarity and recall-to-reject processing. For example, conjunction lure errors could increase because generating cues for the components of parent items increases the familiarity of each component (and therefore the familiarity of later conjunction lures), or because attending to the components of parent items reduces one's ability to recollect a conjunction lure's parents as unitized entities (and thus the ability to employ recall-to-reject processing). Alternatively, this manipulation could reduce conjunction lure errors if elaboratively processing each component of a parent item better enabled participants to use conjunction lures as a cue for recalling parent items on the recognition memory test, enhancing recall-to-reject processing. Despite the ambiguity in expectations for the results of this manipulation, measures of recall-to-reject processing allowed us to assess the impact of component generation on familiarity and recall-to-reject processing. 
As with the first two experiments, the participants came from different countries, and the test instructions and procedures in Experiment 2A were different from those in Experiment 2B. Again recall judgments and actual recall of parent items were collected in Experiment 2B. Also, the experiments differed slightly with regard to the copying of study words. In Experiment 2A, participants copied the study words twice (en masse) in the control condition and did not copy the study word in either of the cue generation conditions. In Experiment 2B, all participants copied the study word once in each study condition, but also supplied cues in the generation conditions. These minor study task differences failed to produce any noticeable differences in the results between Experiments 2A and 2B.

\section{Method}

Participants. Twenty-four students at Victoria University of Wellington participated in Experiment 2A, and 24 students at Middlebury College participated in Experiment 2B as part of an introductory psychology research appreciation requirement.

Materials. The materials and stimuli were the same as those used in Experiments 1A and 1B, except for the sheet given to participants for copying study items and generating cues for study items in the encoding phase of the experiment. The sheet contained either two (Experiment 2A) or three (Experiment 2B), instead of four, lined spaces on each numbered line.

Design and Procedure. A 2 (test item type: parent word vs. offspring) $\times 4$ (study task: copy vs. generate two cues for each parent word vs. generate a cue for each component of a parent word vs. nonstudied) within-participants design was employed. The assignment of stimulus sets to experimental conditions and stimulus counterbalancing procedures were the same as those used in Experiments $1 \mathrm{~A}$ and $1 \mathrm{~B}$. The study instructions and procedure for the study phase were the same as for Experiments 1A and 1B, except for the instructions concerning the writing of cues. In Experiment $2 \mathrm{~A}$, participants were instructed to write the study word twice (e.g., daredevil twice); two cues for the study word (e.g., adventurous, stunt, when presented with daredevil, referred to as the parent generation condition below); or one cue for each of the components of the study word (e.g., truth for the dare component, angel for the devil component when presented with daredevil, referred to as the component generation condition below). In Experiment 2B, participants were instructed to write the study word once (e.g., daredevil); the study word plus two cues for the study word (e.g., daredevil, followed by adventurous and stunt); or the study word plus one cue for each component of the study word (e.g., daredevil, followed by truth for the dare component and angel for the devil component). The test instructions and procedure for the test phase were the same as those for Experiments 1A and 1B, respectively, such that Experiment 2A only required an old-new judgment for each test word, whereas Experiment $2 \mathrm{~B}$ required participants to make an old-new judgment, a recall or know judgment on items judged to be new, and to produce recall of a parent item for items judged to be new due to recall of a parent item.

\section{Results}

In Experiment 2A, the baseline false alarm rates for parents $(M=.06)$ and offspring $(M=.10)$ were similar, but the difference was significant $[t(23)=2.18, S E=$ $.018]$. In Experiment 2B, the baseline false alarm rates for parents $(M=.07)$ and offspring $(M=.07)$ did not differ reliably $[t(23)=0.13]$. Figure 3 depicts the corrected parent-word recognition rates and corrected conjunction lure error rates as a function of study task for Experiment 2A (top panel) and for Experiment 2B (bottom panel). Hits, conjunction lure false alarms, and baselineitem false alarms are presented in Table 4.

The type of study task had a large influence on both parent word recognition and conjunction lure error rates, but it did not affect parent and conjunction lure recognition in the same way. As in Experiments 1A and 1B, generating cues to parent words as a whole considerably enhanced subsequent parent word recognition but produced only a very slight increase in conjunction errors. The generation of cues based on components of parent words also substantially increased parent word recognition in comparison with the copy condition. In fact, generating cues for each component of parent words produced parent-word recognition rates similar to generating cues for parent words as a whole. The component generation task also increased the conjunction lure error rate in comparison with the copy condition and the parent generation condition.
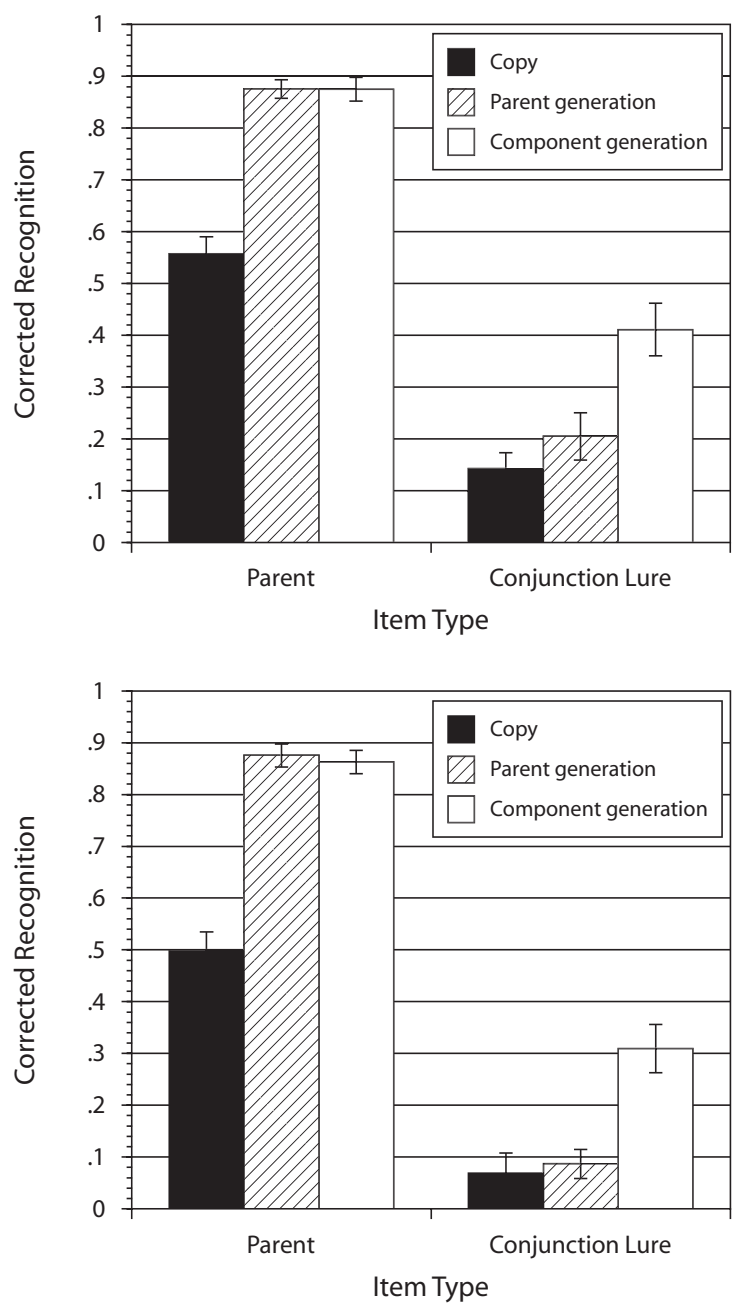

Figure 3. Parent word and conjunction lure recognition, as a function of study task in Experiment 2A (top panel) and Experiment 2B (bottom panel). Error bars depict the standard errors of the means. 
A 3 (study task) $\times 2$ (test item type) repeated measures ANOVA on corrected recognition scores revealed significant interactions in both studies [Experiment 2A, $F(2,46)=11.81, M S_{\mathrm{e}}=.019$; Experiment $2 \mathrm{~B}, F(2,46)=$ 23.76, $\left.M S_{\mathrm{e}}=.017\right]$. In addition, the main effects of study task [Experiment 2A, $F(2,46)=45.27, M S_{\mathrm{e}}=.024$; Experiment $\left.2 \mathrm{~B}, F(2,46)=52.19, M S_{\mathrm{e}}=.022\right]$ and test item type [Experiment $2 \mathrm{~A}, F(1,23)=200.48, M S_{\mathrm{e}}=.048$; Experiment 2B, $\left.F(1,23)=284.78, M S_{\mathrm{e}}=.044\right]$ were significant. To pursue the interaction, we conducted separate repeated measures ANOVAs on parent word recognition and conjunction lure errors, using study task as the independent variable. For parent word recognition, the effect of study task was significant, [Experiment 2A, $F(2,46)=$ 82.64, $M S_{\mathrm{e}}=.010$; Experiment 2B, $F(2,46)=106.60$, $\left.M S_{\mathrm{e}}=.010\right]$. Bonferroni-adjusted comparisons showed that, whereas the generation conditions produced similar levels of parent recognition, both produced higher parentword recognition rates than did the copy condition. As was the case in Experiments 1A and 1B, the very high scores for the generation conditions hamper interpretation of the relative levels of performance in those conditions. The effect of study task was also significant for conjunction lure errors [Experiment 2A, $F(2,46)=14.34, M S_{\mathrm{e}}=.033$; Experiment $\left.2 \mathrm{~B}, F(2,46)=15.40, M S_{\mathrm{e}}=.028\right]$. Bonferroniadjusted comparisons showed that the conjunction lure error rates were higher in the component generation condition than in the two other study conditions, in which they did not differ.

Recall responses. The probability of making a recall response to items for which no competing episodic memory existed was low. Recall responses were rare for parent items (largest $M=.05$ in the copy study condition) as well as for new items that served to measure baseline error rates for both parent items $(M=.04)$ and conjunction lures $(M=.04)$. Furthermore, the conditional probability of making a recall response, given that a test item was called new, was greater than $46 \%$ for lure items, and the conditional probability of making a recall response, given that a test item was called new, was approximately $8 \%$ for old and nonlure new items. Thus, when participants' memory was tested for old or unstudied items, participants largely deemed those items to be new based on lack of familiarity; that is, they gave a know response. In comparison, participants regularly reported using recall in order to reject conjunction lures.

Of central importance is the memorial basis that participants used in judging conjunction lures to be unstudied. The probability of rejecting a conjunction lure due to recall of a parent item (i.e., recall responses) and the probability of correctly recalling a parent item when tested with a conjunction lure are presented in Figure 4. As in Experiment 1B, the ability to produce a parent item to justify a recall response generally supported the validity of recall judgments (Jones, 2005; Jones \& Atchley, 2006). Both measures indicate that participants were able to use recall of a parent item to reject conjunction lures better in the generation conditions than in the copy condition. However, the two generation conditions did not differ in the use of recall-to-reject processing. These observations

\section{Table 4}

Hits for Parent Items, False Alarms for Conjunction Lures, and False Alarms for Baseline Items in Experiments $2 \mathrm{~A}$ and $2 \mathrm{~B}$

\begin{tabular}{|c|c|c|c|c|c|c|c|c|}
\hline & \multicolumn{2}{|c|}{ Copy } & \multicolumn{2}{|c|}{$\begin{array}{c}\text { Parent } \\
\text { Generation }\end{array}$} & \multicolumn{2}{|c|}{$\begin{array}{c}\text { Comp. } \\
\text { Generation } \\
\end{array}$} & \multicolumn{2}{|c|}{ New } \\
\hline & $M$ & $S E$ & $M$ & $S E$ & $M$ & $S E$ & $M$ & $S E$ \\
\hline \multicolumn{9}{|c|}{ Experiment $2 \mathrm{~A}$} \\
\hline Parent & .62 & .04 & .94 & .02 & .94 & .01 & .06 & .01 \\
\hline Conjunction lure & .24 & .04 & .30 & .05 & .51 & .05 & .10 & .02 \\
\hline \multicolumn{9}{|c|}{ Experiment 2B } \\
\hline Parent & .57 & .03 & .95 & .01 & .94 & .02 & .07 & .01 \\
\hline Conjunction lure & .14 & .03 & .16 & .03 & .38 & .05 & .07 & .02 \\
\hline
\end{tabular}

Note-Comp. Generation, component generation.

were supported by a reliable effect of study task [recall judgments, $F(2,46)=11.92, M S_{\mathrm{e}}=.029$; actual parent recall, $\left.F(2,46)=9.41, M S_{\mathrm{e}}=.037\right]$. For both measures, Bonferroni-adjusted comparisons revealed that, whereas the two generation study conditions produced a higher rate of recall-to-reject than did the copy condition, the two generation study conditions did not differ reliably.

Familiarity underlying conjunction errors. As in Experiment 1B, we calculated measures of familiarity based on assumptions of the independence, redundancy, and mutual exclusivity models, using the actual recall rate as a measure of recall-to-reject processing and the conjunction lure false alarm rate as a measure of familiarity without recollection (see Table 5). All three approaches suggested that familiarity was higher in the component generation condition in comparison with the other two encoding conditions [smallest $t(23)=3.78$ ]. However, the three approaches differed with regard to how the parent generation condition and copy condition influenced familiarity in relation to one another. Under redundancy assumptions, cue generation for parents increased familiarity more than the copy condition did $[t(23)=4.96]$, whereas, under the independence $[t(23)=1.48, p>.15]$

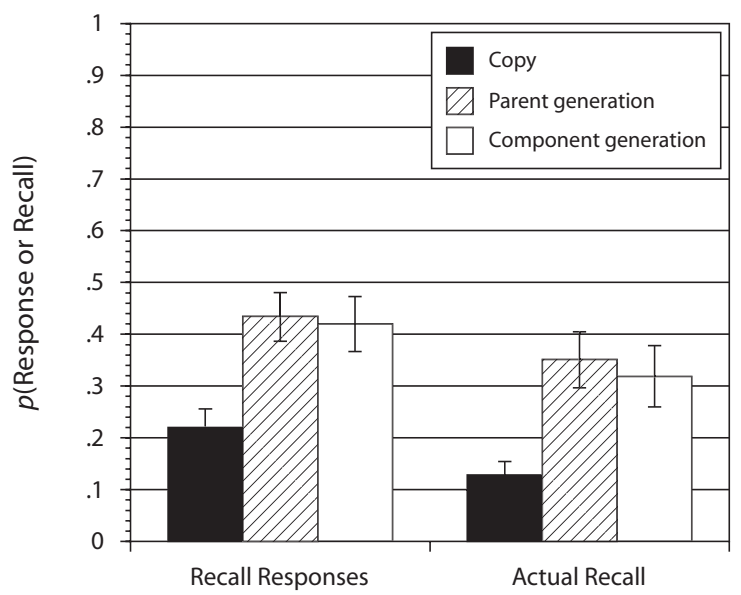

Figure 4. Recall responses and actual recall to conjunction lures judged to be new in Experiment 2B, as a function of study task. Error bars depict the standard errors of the means. 
Table 5

Familiarity Estimates for Experiment 2B, Based on the Assumptions of the Independence, Redundancy, and Mutual-Exclusivity Models

\begin{tabular}{|c|c|c|c|c|c|c|}
\hline \multirow[b]{2}{*}{ Model } & \multicolumn{2}{|c|}{ Copy } & \multicolumn{2}{|c|}{$\begin{array}{c}\text { Generate } \\
2 \text { Cues } \\
\text { (Whole Word) } \\
\end{array}$} & \multicolumn{2}{|c|}{$\begin{array}{c}\text { Generate } \\
2 \text { Cues } \\
\text { (Components) }\end{array}$} \\
\hline & $M$ & $S E$ & $M$ & $S E$ & $M$ & $S E$ \\
\hline Independence & .16 & .04 & .23 & .04 & .51 & .05 \\
\hline Redundancy & .27 & .04 & .51 & .04 & .70 & .03 \\
\hline Mutual exclusivity & .14 & .03 & .16 & .03 & .38 & .05 \\
\hline
\end{tabular}

and mutual exclusivity $[t(23)=0.50, p>.50]$ model assumptions, parent cue generation did not reliably increase familiarity more than the copy condition did. Despite the nonsignificant change in measured familiarity across the copy and parent generation conditions, the independence model did produce a qualitative increase in familiarity of a reasonable magnitude $(M=.07)$, suggesting that the independence model makes claims that are qualitatively consistent with those of the redundancy model.

\section{Discussion}

The results of Experiments 2A and 2B replicate and extend the findings from Experiments $1 \mathrm{~A}$ and $1 \mathrm{~B}$. Both cue-generation study conditions produced larger increases in the hit rates than did the copy control condition. Although a slight, nonsignificant increase in the conjunction error rate was observed for the parent generation condition, a large, significant increase in the conjunction error rate was found for the component generation condition in relation to both the copy condition and cue generation from whole parent words. Thus, the results are generally consistent with the findings of Ghatala et al. (1978) and Odegard et al. (2005). These results also show that the generation of cues at encoding, whether for parent items as a whole or for each component of a compound parent word, increased the participants' ability to use recall-to-reject processing to limit conjunction lure errors. Interestingly, the method of cue generation (for parents vs. components of parents) did not substantially affect the participants' ability to use recall-to-reject processing. What is perhaps most unexpected is that the study condition that produced the highest conjunction error rate also produced a considerable increase in successful recall-to-reject processing in relation to copying parent items during study. Odegard et al. found a similar result, and we take their findings and the present findings to be highly informative. Both results suggest that even when recall-to-reject processing increases across conditions, it is still possible for conjunction lure errors to increase if familiarity is increased by the same condition that improves recall-to-reject processing. This result underscores the importance of collecting measures that separate the influence of recall-to-reject from the influence of familiarity on conjunction lure errors in order to fully understand the processes underlying recognition decisions for conjunction lures. For purposes of comparison, the standard interpretation of an increase in conjunction lure errors from the dual-process perspective would be that familiarity increased without considering the possible effects of the same experimental condition on recall-to-reject processing. Thus, not collecting a measure of recall-to-reject processing would lead one to underestimate how much the condition influenced both familiarity and recall-to-reject processing.

Considering how recall-to-reject processing and the conjunction lure error rates were influenced by the encoding conditions, the redundancy and independence models provide the most reasonable characterization of familiarity processes. Logic similar to that outlined following Experiment 1B suggests that familiarity increased when cues were generated for parent items in comparison with when parent items were copied. Thus, because recall-to-reject processing was higher in the parent generation condition than in the copy condition - but the conjunction lure error rate did not differ across those two conditions-dual-process theories must claim that a second factor (i.e., familiarity) also was higher in the parent generation condition. We compared the general dual-process characterization of the results of Experiment $2 \mathrm{~B}$ with the estimates of familiarity derived from each dual-process model and found that only the views that recollection and familiarity are redundant or are independent processes suggested that familiarity increased with parent generation.

\section{GENERAL DISCUSSION}

The results of these studies provide clear support for dual-process views of conjunction lure errors (Jones \& Atchley, 2002, 2006; Jones \& Jacoby, 2001; Lampinen et al., 2004; Odegard et al., 2005). Specifically, the results of two measures of recall-to-reject processing, coupled with the patterns of conjunction lure errors, provide support for the roles of familiarity and recollection in producing and limiting conjunction lure errors. For example, measures of recall-to-reject processing used in these studies indicated that as the amount of elaborative processing during encoding increased, recall-to-reject processing also increased. This result is consistent with prior work suggesting that the ability to recollect studied items increases with elaborative encoding (H. V. Curran \& Hildebrandt, 1999; Gardiner, 1988; Yonelinas, 2002) and extends the influence of elaborative encoding on recollective processes to memory editing functions (cf. Jones, 2006). Furthermore, the notion that recollection (and therefore recall-to-reject processing) increased with elaborative processing explains the influence of cue generation on parent item recognition, where parent recognition was substantially greater when cue generation occurred than when parents were copied. 
Two results provide evidence of the contribution of familiarity to conjunction lure errors. First, conjunction error rates were always above the baseline false alarm rate, despite test instructions that informed participants of the conjunction lures and evidence that participants often were able to reject conjunction lures based on the retrieval of a parent word. The conjunction effect implies that experimentally induced familiarity kept conjunction lures from being suppressed to levels at or below baseline error levels by memory editing processes (Brainerd, Reyna, \& Kneer, 1995). Second, although levels of recall-to-reject processing were similar when cues were generated for each part of a parent item and for whole parent items (Experiment $2 \mathrm{~B}$ ), conjunction error rates were higher when cues were generated for each part of a parent item (Experiments $2 \mathrm{~A}$ and $2 \mathrm{~B}$ ). Taken together, these two results suggest that a second factor (i.e., familiarity) increased conjunction lure errors more when cues were generated for each part of a parent item. These results reinforce the substantial literature suggesting that conjunction lure errors arise due to recognition memory familiarity (Jones \& Atchley, 2002, 2006; Jones et al., 2007; Jones \& Jacoby, 2001; Lampinen et al., 2004; Odegard et al., 2005; Rubin, Van Petten, Glisky, \& Newburg, 1999).

The collection of objective measures of recollection (i.e., recall-to-reject judgments or recall of parent items when tested with conjunction lures) allowed us to test models of the relationship between recollection and familiarity. On the basis of the general claim of dual-process theory - that recollection and familiarity oppose one another to produce conjunction lure errors-we concluded that property generation for parents increased familiarity (Experiments 1B and 2B) and property generation for the components of parents increased familiarity more than did property generation for parents as whole entities (Experiment 2B). Of the three models we tested (redundancy, independence, mutual exclusivity), only the redundancy model (Joordens \& Merikle, 1993) produced estimates of familiarity that fully aligned with these two conclusions. The independence model (Yonelinas, Dobbins, Szymanski, Dhaliwal, \& King, 1996; Yonelinas \& Jacoby, 1995) produced estimates of familiarity that were qualitatively consistent with the conclusions, but failed to produce an estimate of familiarity's increase with parent generation that was statistically reliable in Experiment 2B. Finally, the mutual exclusivity model (Gardiner, 1988) was inconsistent with the general account offered by dual-process theory, claiming that only component generation in Experiment $2 \mathrm{~B}$ increased familiarity. Thus, the present data favor the conclusion that familiarity and recollection are best viewed as being redundant or independent processes. In addition, although this was not explicitly tested, the data are consistent with the view that recollection and familiarity are correlated, but not entirely redundant, processes (e.g., T. Curran \& Hintzman, 1997).

The conclusion that elaborative encoding increased both recall-to-reject (recollection) and conjunction lure familiarity is consistent with how generation and other types of elaborative processing (e.g., levels of processing) influence recollection and familiarity for studied items.
Yonelinas (2002) conducted an extensive review of studies from which separate measures of recollection and familiarity could be derived. He found that on average, generation and levels of processing encoding manipulations increased estimates of both recollection and familiarity as measured by the independence model. That the same conclusions were reached when the independence model was applied to the present data suggests that measurement models of recollection and familiarity can accurately estimate familiarity supporting conjunction lure errors and that our application of those models to measure familiarity in these studies was appropriate.

One concern readers may have about the claim that conjunction lure error rates were not diminished by parent cue generation is that conjunction error rates were relatively low (generally less than .15 above baseline in the copy condition). There are three reasons why we believe that these low overall error rates, sometimes observed in the conjunction lure paradigm, do not compromise the interpretation that elaborative encoding failed to decrease conjunction lure errors. First, the pattern of means in all four experiments suggests that generating cues for whole parent items increased conjunction lure errors, even though none of the mean differences was statistically reliable (see Figures 1 and 3). Second, evidence exists that it is possible for recall-to-reject processing to suppress lure false alarm rates below the false alarm rate to unstudied and unrelated test foils (Brainerd et al., 1995). Thus, the range of recognition scores between the corrected recognition score for conjunction lures in the copy condition and zero does not represent the entire plausible range for corrected conjunction lure recognition scores. Instead, the plausible range for corrected conjunction lure recognition scores extends below zero, so that suppression of conjunction lure false alarms with recall-to-reject processing could have produced scores as low as -.10 in Experiments 1B and $2 \mathrm{~A},-.06$ in Experiment $1 \mathrm{~A}$, and -.07 in Experiment $2 \mathrm{~B}$, where the lower bound of the plausible range of conjunction lure recognition scores is represented by the false alarm rate to baseline items for a given experiment subtracted from zero. Indeed, the false alarm rates reported by Brainerd et al. (1995) were exceedingly low when recall-to-reject processing was used (typically less than .10 ), whereas the baseline false alarm rates were considerably higher (typically greater than .20 ), suggesting that recall-to-reject processing can produce dramatic decreases in false alarm rates, even though it did not in the present studies. Third, we observed a substantial increase in the use of recall-to-reject processing between the copy condition $(M=.24)$ and the three-cues-generated condition $(M=$ $.56)$ in Experiment 1B. Despite this very large increase in recall-to-reject processing, conjunction lure error scores were actually slightly higher in the three-cues-generated condition $(M=.09)$ than in the copy condition $(M=.07)$. Taken together, these three factors suggest that it is unlikely that floor effects compromised the ability of these studies to observe a decrease in conjunction lure errors.

A second concern readers may have about these results is the use of recall judgments to measure recall-to-reject processing. In particular, our use of phenomenological 
judgments to measure recall-to-reject processing may cause concern due to the debate over the proper interpretation of remember and know judgments for items judged to be old (e.g., Donaldson, 1996; Dunn, 2004; Gardiner, Richardson-Klavehn, \& Ramponi, 1998; Hirshman, 1998; Jacoby, Jones, \& Dolan, 1998; Yonelinas, 2002). There are three primary reasons we believe that recall judgments provide a valid measure of strategic retrieval processes, thereby making our dual-process interpretation of the present data appropriate. First, in this case, recollection is defined at the item level as retrieval of a parent word, not as an unverifiable thought that a participant claims to have had during the study phase. Second, we verified participants' recall judgments by asking them to produce the parent item that led them to make a recall judgment. In both experiments, actual recall of parent items corresponded closely with recall judgments to conjunction lure items across all of the encoding conditions. Third, participants showed considerable selectivity in their use of recall judgments. When participants judged a test item to be new, it was accompanied by a recall judgment less than $10 \%$ of the time if the test item was studied or was a new item used to measure baseline error rates. In contrast, conjunction lures judged to be new were accompanied by a recall judgment nearly $50 \%$ of the time. Thus, when a test item was judged to be new and could not have been rejected on the basis of recall of a studied item (i.e., it was a baseline item or a studied item), participants overwhelmingly claimed that the item was unfamiliar. However, when a test item was judged to be new and it was possible to use recall of a studied item as a basis for the new judgment (i.e., the test item was a conjunction lure), participants regularly reported using recall-to-reject processing.

To this point, we have emphasized an explanation of these data offered by dual-process theories of recognition memory. However, in the literature on conjunction lure errors, there is a second view: feature-configuration or binding theory (Reinitz et al., 1996). In its original form, which is more applicable to the present data, binding theory proposes that conjunction lure errors occur when participants fail to bind together the features (component words) of a parent item at encoding, leading each feature to be stored separately. When a conjunction lure is presented during retrieval, the features in the conjunction lure are sometimes conjointly retrieved, leading participants to judge the lure to have been studied.

At a general level, the binding account is consistent with the dual-process approach, in that errors can be avoided via the retrieval of a configuration from the study episode (i.e., a parent item). However, in binding theory it is assumed that if a configuration is stored, no error will be made for its corresponding conjunction lure (Reinitz et al., 1996; for criticism of this assumption, see Jones \& Jacoby, 2001, and note 1 of Jones \& Atchley, 2002). Thus, factors that boost memory for configurations should increase hits but decrease conjunction errors, and factors that hurt memory for configurations should decrease hits but increase conjunction errors.

The binding account can explain some aspects of the present data. Specifically, conditions that produced higher hit rates, presumably due to the better storage of configurations, also produced higher rates of recall responses. Despite this general support for binding theory, however, several elements of these data are not easily explained by binding theory. For example, it is not clear why conjunction error rates were unaffected by parent generation if configurations were stored better in some conditions than in others, as indexed by changes in hit rates and recall responses. More problematic are the data for the component generation condition in Experiments 2A and 2B. A focus on the components of a parent word during encoding should discourage the binding of the two features of a parent item, leading to an increase in conjunction errors but a decrease in recall responses and, likely, in hits. Yet, the component generation condition increased the hit, conjunction error, and recall response rates in relation to the copy condition. Finally, the component generation condition increased conjunction error rates in relation to the parent generation condition, despite similar hit and recall response rates in those two conditions. Thus, binding theory's assumption that configuration storage increases hits and decreases conjunction lure errors appears to be too restrictive to explain the present data.

A second alternative to dual-process theory's explanation of the present data is that recall of the components of parent items may have produced some conjunction lure errors. Were this the case, conjunction lure error rates would have been driven by a combination of familiarity and recalled components, and the dual-process assumptions we used to derive estimates of familiarity would not have been accurate. This hypothesis is similar to the concern that nondiagnostic or noncriterial recollection influences estimates of familiarity in the process-dissociation procedure (Mulligan \& Hirshman, 1997; Yonelinas \& Jacoby, 1996). Thus, since our task only measured recollection as recall-to-reject processing, it is not possible to know whether, or the extent to which, recollection may have increased conjunction lure errors. As with noncriterial recollection's potential effect on process-dissociation estimates of familiarity, had recall contributed to conjunction lure errors, it would have been measured erroneously as familiarity by our estimation procedures.

One way to evaluate whether component recall invalidates the dual-process claims we have made is to assess whether a model assuming only component recall and recall-to-reject can accommodate our data. In essence, by testing this restricted theory, we can assess the necessity of positing familiarity differences across experimental conditions in order to explain conjunction lure errors. This theory can explain the results from the copy condition and the component generation conditions by proposing that component generation inspires greater component recall, producing more conjunction lure errors, and greater parent recall, producing higher rates of recall-to-reject and hits. However, this theory is unable to explain why generating properties for whole parent items did not decrease conjunction lure errors. Specifically, there is no reason to believe that parent generation would increase recall of conjunction lure components more than copying parent words would. Thus, because the data suggest that parent generation in- 
creased recall-to-reject processing in comparison with the copy condition, this theory would expect a decrease in conjunction lure errors when parents are generated in relation to when parents are copied - a result that was not observed in any of our experiments. We take this erroneous prediction to indicate that a component recall process does not sufficiently explain the conjunction lure data from these studies, and that a familiarity process is necessary to explain conjunction lure errors. Although it is plausible that recall-based processes may contribute to conjunction lure false alarm rates, parsimony considerations favor the standard dual-process theory because it can explain all of the data without positing recall-based conjunction lure errors.

Despite the data favoring dual-process theory in general, it is important to realize that to the extent that component recall contributes to conjunction lure errors, our estimates of familiarity would be in error. However, even if some conjunction lure errors were due to component recall, it is not necessarily the case that component recall occurred sufficiently often to change the conclusions that familiarity increased following parent generation and that familiarity increased to a greater extent following component generation than following parent generation. Furthermore, the possible contribution of component recall to conjunction lure errors does not undermine the other primary conclusions from these studies - that recall-toreject processing increased following generation of both whole parent items and components of parent items, and that these increases in recall-to-reject processing did not reduce conjunction lure error rates.

\section{Conclusion}

In closing, we wish to emphasize the utility of measures of recall-to-reject processing for understanding recognition memory errors and the contribution of recollection and familiarity to conjunction lure errors. The collection of such measures allows precise examination of the bases of recognition memory errors, and, therefore, the extent to which participants use strategic processes to limit conjunction lure errors. Furthermore, the collection of recallto-reject measures allows one to compute a measure of familiarity for conjunction lures that is based on an objective, verifiable measure of recollection, mollifying common concerns about the use of recollection judgments as a measure of recollection (e.g., Donaldson, 1996; Dunn, 2004). Finally, collecting recall-to-reject measures would appear to be useful in other paradigms as well, such as plurality discrimination and associative recognition, in which recall-to-reject processing is encouraged and perhaps likely to occur. Indeed, given that the evidence for recall-to-reject in the plurality and associative recognition paradigms is sometimes open to multiple interpretations (e.g., Rotello \& Heit, 1999, 2000), the addition of another measure of recall-to-reject processing could prove to be a useful tool for understanding the contribution of recollection and familiarity to recognition of similar test lures.

\section{AUTHOR NOTE}

Correspondence concerning this article should be sent to J. Arndt, Department of Psychology, Middlebury College, Middlebury, VT
05753 (e-mail: jarndt@middlebury.edu) or T. Jones, School of Psychology, Victoria University of Wellington, P.O. Box 600, Wellington, New Zealand (e-mail: todd.jones@vuw.ac.nz).

\section{REFERENCES}

Brainerd, C. J., Reyna, V. F., \& Kneer, R. (1995). False-recognition reversal: When similarity is distinctive. Journal of Memory \& Language, 34, 157-185.

Brainerd, C. J., Reyna, V. F., Wright, R., \& Mojardin, A. H. (2003). Recollection rejection: False-memory editing in children and adults. Psychological Review, 110, 762-784.

Curran, H. V., \& Hildebrandt, M. (1999). Dissociative effects of alcohol on recollective experience. Consciousness \& Cognition, 8 , 497-509.

Curran, T., \& Hintzman, D. L. (1997). Consequences and causes of correlations in process dissociation. Journal of Experimental Psychology: Learning, Memory, \& Cognition, 23, 496-504.

Diana, R., Reder, L. M., Arndt, J., \& Park, H. (2006). Models of recognition: A review of arguments in favor of a dual-process account. Psychonomic Bulletin \& Review, 13, 1-21.

DonalDSON, W. (1996). The role of decision processes in remembering and knowing. Memory \& Cognition, 24, 523-533.

DunN, J. C. (2004). Remember-know: A matter of confidence. Psychological Review, 111, 524-542.

GALLO, D. A. (2004). Using recall to reduce false recognition: Diagnostic and disqualifying monitoring. Journal of Experimental Psychology: Learning, Memory, \& Cognition, 30, 120-128.

GARDINER, J. M. (1988). Functional aspects of recollective experience. Memory \& Cognition, 16, 309-313.

Gardiner, J. M., Richardson-Klavehn, A., \& Ramponi, C. (1998). Limitations of the signal detection model of the remember-know paradigm: A reply to Hirshman. Consciousness \& Cognition, 7, 285-288.

Ghatala, E. S., Levin, J. R., Bell, J. A., Truman, D. L., \& Lodico, M. G. (1978). The effect of semantic and nonsemantic factors on the integration of verbal units in recognition memory. Journal of Experimental Psychology: Human Learning \& Memory, 4, 647-655.

Hintzman, D. L., Curran, T., \& OpPY, B. (1992). Effects of similarity and repetition on memory: Registration without learning? Journal of Experimental Psychology: Learning, Memory, \& Cognition, 18, 667-680.

HiRSHMAN, E. (1998). On the utility of the signal detection model of the remember-know paradigm. Consciousness \& Cognition, 7, 103-107.

JACOBY, L. L. (1991). A process dissociation framework: Separating automatic from intentional uses of memory. Journal of Memory \& Language, 30, 513-541.

JaCoBY, L. L., Jones, T. C., \& Dolan, P. O. (1998). Two effects of repetition: Support for a dual-process model of knowledge judgments and exclusion errors. Psychonomic Bulletin \& Review, 5, 705-709.

JONES, T. C. (2005). Study repetition and the rejection of conjunction lures. Memory, 13, 499-515.

JoNES, T. C. (2006). Editing (out) generated study words in a recognition exclusion task: Effects of response signal delay and generation procedure. Memory, 14, 712-729.

JoNes, T. C., \& ATCHLEY, P. (2002). Conjunction error rates on a continuous recognition memory test: Little evidence for recollection. Journal of Experimental Psychology: Learning, Memory, \& Cognition, 28, 374-379.

Jones, T. C., \& ATchley, P. (2006). Conjunction errors, recollectionbased rejections, and forgetting in a continuous recognition task. Journal of Experimental Psychology: Learning, Memory, \& Cognition, 32, 70-78.

Jones, T. C., Brown, A. S., \& ATChLEY, P. (2007). Feature and conjunction effects in recognition memory: Toward specifying familiarity for compound words. Memory \& Cognition, 35, 984-998.

JONES, T. C., \& JACOBY, L. L. (2001). Feature and conjunction errors in recognition memory: Evidence for dual-process theory. Journal of Memory \& Language, 45, 82-102.

JoORdens, S., \& MeriKLe, P. M. (1993). Independence or redundancy? Two models of conscious and unconscious influences. Journal of Experimental Psychology: General, 122, 462-467.

Lampinen, J. M., Odegard, T. N., \& Neuschatz, J. S. (2004). Robust recollection rejection in the memory conjunction paradigm. Journal 
of Experimental Psychology: Learning, Memory, \& Cognition, 30, 332-342.

MäNTYLÄ, T. (1986). Optimizing cue effectiveness: Recall of 500 and 600 incidentally learned words. Journal of Experimental Psychology: Learning, Memory, \& Cognition, 12, 66-71.

MänTYlë, T., \& Nilsson, L.-G. (1983). Are my cues better than your cues? Uniqueness and reconstruction as prerequisites for optimal recall of verbal materials. Scandinavian Journal of Psychology, 24, 303-312.

Mulligan, N. W., \& Hirshman, E. (1997). Measuring the bases of recognition memory: An investigation of the process dissociation framework. Journal of Experimental Psychology: Learning, Memory, \& Cognition, 23, 280-304.

Odegard, T. N., Lampinen, J. M., \& Toglia, M. P. (2005). Meaning's moderating effect on recollection rejection. Journal of Memory \& Language, 53, 416-429.

Reder, L. M., Nhouyvanisvong, A., Schunn, C. D., Ayers, M. S., Angstadt, P., \& Hiraki, K. (2000). A mechanistic account of the mirror effect for word frequency: A computational model of remember-know judgments in a continuous recognition paradigm. Journal of Experimental Psychology: Learning, Memory, \& Cognition, 26, 294-320.

Reinitz, M. T., Lammers, W. J., \& Cochran, B. P. (1992). Memoryconjunction errors: Miscombination of stored stimulus features can produce illusions of memory. Memory \& Cognition, 20, 1-11.

Reinitz, M. T., Verfaellie, M., \& Milberg, W. P. (1996). Memory conjunction errors in normal and amnesic subjects. Journal of Memory \& Language, 35, 286-299.

Roediger, H. L., III, McDermott, K. B., \& Robinson, K. J. (1998). The role of associative processes in producing false memories. In M. A. Conway, S. E. Gathercole, \& C. Cornoldi (Eds.), Theories of memory (Vol. 2, pp. 187-245). Hove, U.K.: Psychology Press.

Rotello, C. M., \& Heit, E. (1999). Two-process models of recognition memory: Evidence for recall-to-reject? Journal of Memory \& Language, 40, 432-453.

Rotello, C. M., \& HeIt, E. (2000). Associative recognition: A case of recall-to-reject processing. Memory \& Cognition, 28, 907-922.

Rotello, C. M., Macmillan, N. A., \& Van Tassell, G. (2000). Recall-to-reject in recognition: Evidence from ROC curves. Journal of Memory \& Language, 43, 67-88.

Rubin, S. R., Van Petten, C., Glisky, E. L., \& Newburg, W. M. (1999). Memory conjunction errors in younger and older adults:
Event-related potential and neuropsychological data. Cognitive Neuropsychology, 16, 459-488.

Schneider, W., Eschman, A., \& Zuccolotto, A. (2002). E-Prime user's guide. Pittsburgh: Psychology Software Tools.

Underwood, B. J., KapelaK, S. M., \& Malmi, R. A. (1976). Integration of discrete verbal units in recognition memory. Journal of Experimental Psychology: Human Learning \& Memory, 2, 293-300.

Underwood, B. J., \& Zimmerman, J. (1973). The syllable as a source of error in multisyllable word recognition. Journal of Verbal Learning \& Verbal Behavior, 12, 701-706.

YonelinAs, A. P. (2002). The nature of recollection and familiarity: A review of 30 years of research. Journal of Memory \& Language, $\mathbf{4 6}$, 441-517.

Yonelinas, A. P., Dobbins, I., Szymanski, M. D., Dhaliwal, H. S., \& KING, L. (1996). Signal-detection, threshold, and dual-process models of recognition memory: ROCs and conscious recollection. Consciousness \& Cognition, 5, 418-441.

Yonelinas, A. P., \& JACOBY, L. L. (1995). The relationship between remembering and knowing as bases for recognition: Effects of size congruency. Journal of Memory \& Language, 34, 622-643.

Yonelinas, A. P., \& JACOBY, L. L. (1996). Noncriterial recollection: Familiarity as automatic, irrelevant recollection. Consciousness \& Cognition, 5, 131-141.

\section{NOTES}

1. The claim here is not that recollection is available earlier in retrieval than familiarity is, but that when both processes are available, as is typically the case in recognition memory experiments using untimed testing conditions, participants will preferentially use recollection.

2. Data from all four experiments were also analyzed using $d^{\prime}$ and $A^{\prime}$ to correct hits and conjunction lure false alarms for baseline false alarm rates. These analyses led to the same conclusions as those using corrected recognition scores, with one exception: The $A^{\prime}$ analyses of Experiment $1 \mathrm{~A}$ did not produce a significant interaction between study task and test item type $[F(2,46)=2.72, p<.08]$. Despite the interaction's only approaching significance, Bonferroni-adjusted comparisons showed the same patterns of performance that were found with analyses of corrected recognition and $d^{\prime}$

(Manuscript received May 14, 2007; revision accepted for publication January 14, 2008.) 